\title{
A Study of Fuzzy Ideals in BCK Algebra
}

\author{
M. Alcheikh ${ }^{1} \&$ Anas Sabouh ${ }^{1}$ \\ ${ }^{1}$ Faculty of Science, Department of Mathematics, Idlib University, Syria \\ Correspondence: Anas Sabouh, Faculty of Science, Department of Mathematics, Idlib University, Syria.
}

Received: August 7, 2019 Accepted: September 2, 2019 Online Published: September 9, 2019

doi:10.5539/jmr.v11n5p11

URL: https://doi.org/10.5539/jmr.v11n5p11

\section{Abstract}

In this paper, some fuzzy ideals in BCK-Algebra has been discussed and some theorems relating with fuzzy ideals and anti-fuzzy ideals in BCK-Algebra have been proved.

Keywords: BCK Algebra, ideal, fuzzy ideal, anti-Fuzzy ideal, anti-fuzzy p-ideal

\section{Introduction}

The study of BCK-algebra was initiated by Imai and Is'eki (IMAI\& ISKI, 1966) in 1966 as a generalization of the concept of set-theoretic difference and propositional calculi. The concept of fuzzy sets was introduced by L. A. Zadeh (ZADEH, 1965). And Rosenfeld (AZRIEL ROSENFELD, 1971) The first to apply the concept of fuzzy sets to algebraic systems in 1971. And O.Xi applied the concept of fuzzy sets on BCK-algebras.

\section{Preliminaries}

Definition 2.1. (Meng \& Guo, 2005) Let $(X, *, 0)$ be a groupoid with a distinguished element 0 and a binary operation*. Then $(X, *, 0)$ is a BCI-algebra if: for all $x, y, z \in X$,

(I) $((x * y) *(x * z)) *(z * y)=0$,

(II) $(x *(x * y)) * y=0$,

(III) $x * x=0$,

(IV) $x * y=0$ and $y * x=0$ imply $x=y$

In a BCI-algebra $\mathrm{X}$, a partially ordered relation $\leq$ can be deIned by

$x \leq y$ If and only if $x * y=0$

A BCI-algebra is said to be a BCK-algebra if it satisfies:

(IIV) $0 * x=0$ for all $x \in X$.

A BCK/BCI-algebra $X$ has the following properties: (Jun\& Lee, 2011)

(b1) $(\forall x \in X)(x * 0=x)$.

(b2) $(\forall x, y, z \in X)((x * y) * z=(x * z) * y)$.

(b3) $(\forall x, y, z \in X)(x \leq y \Rightarrow x * z \leq y * z, z * y \leq z * x)$.

(b4) $(\forall x, y, z \in X)((x * z) *(y * z) \leq x * y)$.

In particular, if $\mathrm{X}$ is a $\mathrm{BCK}$-algebra then the following property hold:

(b5) $(\forall x, y \in X)((x * y) * x=0)$.

Taking the set $A=\{0, a, b\}$ combined with the operator* defined in the table below

\begin{tabular}{c|ccc}
\hline$*$ & 0 & $a$ & $b$ \\
\hline 0 & 0 & $b$ & $a$ \\
$a$ & $a$ & 0 & $b$ \\
$b$ & $b$ & $a$ & 0 \\
\hline
\end{tabular}


It is clear that BCI-algebra axioms are satisfied, so $A$ is BCI-algebra, but is not BCK-algebra, notice that $0 * a \neq 0$ from the table means that Axiom (IIV) is not satisfied from BCK-algebra.

Definition 2.2. (Zahedi\& Bozorgee, 1999) A BCK-algebra $X$ is said to be bounded if there exists an element $1 \in X$ such that $x \leq 1$ for all $x \in X$.

We will denote $1 * x$ by $N x$ for brief. We note that $N 1=0$ and $N 0=1$ in a bounded BCK-Algebra.

Definition 2.3. (Zahedi\& Bozorgee, 1999) A BCK-algebra X is called commutative if

$$
x *(x * y)=y *(y * x) \text { For all } x, y \in X
$$

Definition 2.4. (Ahsan \& Deeba, 1989) A BCK algebra X is called implicative if

$$
x *(y * x)=x \text { for all } x, y \in X
$$

Definition 2.5. (Jun, \& Öztürk, 2018) A BCK-algebra X is positive implicative if it satisfies

the following condition:

$$
(\forall x, y, z \in X)((x * z) *(y * z)=(x * y) * z) .
$$

Definition 2.6. (Meng \& Guo, 2005) A nonempty subset I of a BCK/BCI-algebra X is said to be an ideal if it satisfies:

(I1) $0 \in I$, and (I2) for all $x, y \in X ; x * y \in I$ and $y \in I$ imply $x \in I$.

Any ideal I has the property: (I3) $x \in I$ and $y \leq x$ imply y $\in \mathrm{I}$

Definition 2.7. (Kordi\& Moussavi, 2007) Let $\mathrm{X}$ be a set. A fuzzy set $\mu$ in $\mathrm{X}$ is a function

$$
\mu: \mathrm{X} \rightarrow[0,1] \text {. }
$$

Definition 2.8. (Kordi\& Moussavi, 2007) A fuzzy set $\mu$ in a BCI-algebra $\mathrm{X}$ is said to be a fuzzy ideal in $\mathrm{X}$ if it satisfies:

(F1) $\mu(0) \geq \mu(x)$,

(F2) $\mu(x) \geq \min \{\mu(x * y), \mu(y)\}$ for all $x, y \in X$.

If $\mu$ is fuzzy ideal in $X$ then, for $x, y, z \in X$, such that $(x * y) * z=0$, then

$$
\mu(x) \geq \min \{\mu(y), \mu(z)\}
$$

Definition 2.9. (Hong \& Jun, 1998)A fuzzy subset $\mu$ of a BCK-algebra $X$ is called an anti-fuzzy ideal of $X$ if

(i) $\mu(0) \leq \mu(x)$,

(ii) $\mu(x) \leq \max \{\mu(x * y), \mu(y)\}$,

For all $x, y \in X$.

Definition 2.10 (Kordi\& Moussavi, 2007) A nonempty subset $A$ in a BCI-algebra $X$ is called a p-ideal of $X$ if it satisfies

(i1) $0 \in A$,

(i2) If for all $x, y, z \in X,(x * z) *(y * z) \in A$ and $y \in A$, imply that $x \in A$.

If we put $z=0$, then it follows that $\mathrm{A}$ is an ideal. Thus, every p-ideal is an ideal.

Definition 2.11. (Kordi\& Moussavi, 2007) Let $\mathrm{X}$ be a BCI-algebra. A fuzzy subset $\mu$ in $\mathrm{X}$ is called a fuzzy p-ideal if it satisfies

(F1) $\mu(0) \geq \mu(x)$,

(F2) $\mu(x) \geq \min \{\mu((x * z) *(y * z)), \mu(y)\}$, for all $x, y, z \in X$.

Definition 2.12. (Mostafa, Omar \& Ahmed, 2011) A fuzzy set $\mu$ in $X$ is called anti fuzzy p-ideal of $\mathrm{X}$ if it satisfies:

(AF1) $\mu(0) \leq \mu(x)$

(AF5) $\mu(x) \leq \max \{\mu((x * z) *(y * z)), \mu(y)\}$ for all $x, y, z \in X$

Definition 2.13. (Solairaju \& Ragavan , 2011) A fuzzy ideal $\mu$ in a BCI-algebra $\mathrm{X}$ is said to be closed if for all $x \in$ $X, \mu(0 * x) \geq \mu(x)$. 
Theorem 2.14. (Meng \& Guo, 2005) Let $\mu$ be a fuzzy ideal in a BCI-algebra X. Then $\mu$ is closed if and only if for any $x, y \in X, \mu(x * y) \geq \mu(x) \wedge \mu(y)$, that is, $\mu$ is a fuzzy subalgebra in $\mathrm{X}$.

\section{Results}

In this paper, we defined ideals generated by a set in finite and implicative BCK-Algebra, and main ideal generated by element in implicative BCK Algebra.

We found also equivalence definition for fuzzy ideals and anti-fuzzy ideals in BCK Algebra. We changed fuzzy ideals into fuzzy sub-Algebra of BCK-Algebra, and we proved in implicative BCI Algebra that the fuzzy set is anti- fuzzy p-ideal in BCI-Algebra if and only if the fuzzy set is anti-fuzzy ideal within certain conditions.

Theorem 3.1. Let $I$ be a ideal in a BCK-algebra X, $x \in I$ then

$$
x * y \in I \quad ; \forall y \in I
$$

Proof. Since $x * y \leq x$ for all $x, y \in X$,then $(x * y) * x=0$, And since $\mathrm{I}$ is an ideal, so $0 \in I$, hence $(x * y) * x \in I$, but $x \in I$, so by the definition of ideal we get $x * y \in I$.

Theorem 3.2. Let $\mathrm{X}$ be implicative and finite $\mathrm{BCK}$ algebra, and $\emptyset \neq \mathrm{S} \subseteq \mathrm{X}$ then, the set:

$$
A=\left\{x \in X ; x \leq x_{1} \vee x_{2} \vee \ldots \vee x_{n} ; x_{i} \in S \quad, i=1,2, \ldots, n ; n \in Z^{+}\right\}
$$

is ideal in $\mathrm{X}$ contains $\mathrm{S}$, and it is the smallest ideal which contains $\mathrm{S}$.

Proof. Since $0 * x=0$ for all $x \in X$, then $0 *\left(x_{1} \vee x_{2} \vee \ldots \vee x_{n}\right)=0$

$\Rightarrow 0 \leq x_{1} \vee x_{2} \vee \ldots \vee x_{n} \quad \Rightarrow 0 \in \mathrm{A}$

If $x * y \in A \quad \& \quad y \in A$ then $x * y \leq x_{1} \vee x_{2} \vee \ldots \vee x_{n} \& \mathrm{y} \leq x_{1} \vee x_{2} \vee \ldots \vee x_{n}$

$\Longrightarrow(x * y) *\left(x_{1} \vee x_{2} \vee \ldots \vee x_{n}\right)=0 \& \mathrm{y} *\left(x_{1} \vee x_{2} \vee \ldots \vee x_{n}\right)=0$

And by the definition 2.5. We get

$\left(x *\left(x_{1} \vee x_{2} \vee \ldots \vee x_{n}\right)\right) *\left(y *\left(x_{1} \vee x_{2} \vee \ldots \vee x_{n}\right)\right)=0$

$\Longrightarrow\left(x *\left(x_{1} \vee x_{2} \vee \ldots \vee x_{n}\right)\right) * 0=0$

$\Rightarrow\left(x *\left(x_{1} \vee x_{2} \vee \ldots \vee x_{n}\right)\right) * 0=0$

$\Rightarrow x *\left(x_{1} \vee x_{2} \vee \ldots \vee x_{n}\right)=0$

$\Rightarrow x \leq x_{1} \vee x_{2} \vee \ldots \vee x_{n}$

$\Rightarrow \quad x \in A$

So, we conclude the $\mathrm{A}$ is ideal in $\mathrm{X}$.

If $\mathrm{B}$ is another ideal in $\mathrm{X}$ contains $\mathrm{S}$, then

$\forall x \in A \Rightarrow x \in X ; x \leq x_{1} \vee x_{2} \vee \ldots \vee x_{n} ; x_{i} \in S \subseteq B \quad, i=1,2, \ldots, n$

$\Rightarrow x_{1} \vee x_{2} \vee \ldots \vee x_{n} \in B \quad \Rightarrow x \in B \quad \Rightarrow A \subseteq B$

We conclude that $\mathrm{A}$ is ideal in $\mathrm{X}$ contains $\mathrm{S}$ and it's the smallest ideal in $\mathrm{X}$ contain $\mathrm{S}$.

Theorem 3.3. Let $X$ be implicative and finite BCK algebra and let $a \in X$, then the set $A=\{x \in X ; x \leq a\}$ is an ideal in X contains $a$ and it's the smallest ideal in X contains $a$.

Proof. $0 \leq a \Rightarrow 0 \in A$ If $x * y \in A \& y \in A$ then $x * y \leq a \& y \leq a$

$\Rightarrow x * y \leq a \Rightarrow(x * y) * a=0$ In addition, by the definition 2.5. We get

$\Rightarrow y * a=0 \quad(x * a) *(y * a)=0$, But $y \leq a$

$\Longrightarrow(x * a) * 0=0 \Longrightarrow x * a=0$

$\Rightarrow x \leq a \Rightarrow x \in A$

Therefore, we conclude the A is ideal in $\mathrm{X}$.

If $\mathrm{B}$ is another ideal in $\mathrm{X}$ contains $a$, then $\forall x \in A \Rightarrow x \leq a \Rightarrow x \in B$

So $A \subseteq B$. We conclude that $\mathrm{A}$ is ideal in X contains $a$ and it is the smallest ideal in X contain $a$.

Definition 3.4. Let $X$ be implicative and finite BCK algebra and let $a \in X$, then we call the ideal exists in theorem (3.2.) with main ideal generated by element $a$, and we denote it by $\langle a\rangle$, so

$$
<\mathrm{a}>=\{x \in X ; x \leq a\}
$$


Notice 3.5. The two theorems (3.1.) and (3.2.) are not satisfied in BCI algebra.

Theorem 3.6. Let $\mathrm{X}$ be $\mathrm{BCK}$ algebra and let $\mu$ be fuzzy set in $\mathrm{X}$, then $\mu$ is anti-fuzzy ideal in $\mathrm{X}$ if and only if for any element $a \in[0,1]$ then the next set is ideal in $\mathrm{X}$.

$$
A_{a}=\{x \in X \quad ; \mu(x) \leq a\} \quad ; A_{a} \neq \varnothing
$$

Proof. Suppose that $\mu$ is anti-fuzzy ideal in $\mathrm{X}$, and since for $x \in A_{a}$ Take $\mathrm{t} \in[0,1]$ such that $A_{a} \neq \emptyset$ and let $x, y \in X$ such that $x \in A_{a}$. Then $\mu(x) \leq a$, and also we have $\mu$ is anti-fuzzy ideal in $\mathrm{X}$, so $\mu(0) \leq \mu(x)$

$\Rightarrow \mu(0) \leq \mu(x) \leq a \Rightarrow \mu(0) \leq a \Rightarrow 0 \in A_{a}$

Let $x * y \in A_{a} \& y \in A_{a}$

We have $x * y \in A_{a} \Rightarrow \mu(x * y) \leq a$

And $y \in A_{a} \Rightarrow \mu(y) \leq a$, we have $\mu$ is anti-fuzzy ideal in X, so

$\mu(x) \leq \mu(x * y) \wedge \mu(y) \leq a \wedge a=a$

$\Rightarrow \mu(x) \leq a \quad \Rightarrow \quad x \in A_{a}$ Therefore, $A_{a}$ is an ideal in X.

Now suppose for contradiction that $\mu$ is not an anti-fuzzy ideal of $\mathrm{X}$.

If (i) from 2.9. is not true, that is $\mu(0)>\mu(x)$, for some $x \in X$ then we take

$a_{1}=\frac{1}{2}(\mu(0)+\mu(x))$, so $\mu(0)>a_{1} \& \mu(x)<a_{1}$, hence

$0 \leq \mu(x)<a_{1} \leq 1$, therefore $x \in A_{a}$, which means that $A_{a_{1}} \neq \emptyset$.

As $A_{a}$ is an ideal we have $0 \in A_{a_{1}}$ implies $\mu(0) \leq a_{1}$, which is a contradiction because $\mu(0)>a_{1}$, therefore $\mu(0) \leq$ $\mu(x)$ for all $x \in X$.

If $\mu(x)>\mu(x * y) \vee \mu(y)$, we take $a_{2}=\frac{1}{2}(\mu(x)+(\mu(x * y) \vee \mu(y))$, then

$(\mu(x * y) \vee \mu(y))<a_{2}<\mu(x)$, so $x \notin A_{a_{2}}$ which gives a contradiction by (I2) from 2.6. Therefore

$\mu(x) \leq \mu(x * y) \vee \mu(y)$.

Theorem 3.7. Let $\mathrm{X}$ be BCK algebra and let $\mu$ be fuzzy set in $\mathrm{X}$, if

$(x * a) *(y * b)=(x * y) *(a * b) \quad \forall x, y, a, b \in X$, then

1. $\mu(x * y)=\mu(y * x), \forall x, y \in X$

2. $\mu$ is fuzzy sub-algebra of $\mathrm{X}$.

Proof. 1- we have $\mu(x * y)=\mu((x * y) * 0)$, and by (III) from 2.1.

$$
\begin{array}{r}
\mu(x * y)=\mu((x * y) *(x * x)), \text { and by the assumption } \\
\mu(x * y)=\mu((x * x) *(y * x)) \\
\mu(x * y)=\mu(0 *(y * x))=\mu(0) \geq \mu(y * x), \text { therefore }
\end{array}
$$

$\mu(x * y) \geq \mu(y * x)$

Also $\mu(y * x)=\mu((y * x) * 0)$, and by (III) from 2.1.

$\mu(y * x)=\mu((y * x) *(y * y))$, and by the assumption

$$
\mu(y * x)=\mu((y * y) *(x * y))=\mu(0 *(x * y))
$$

$\mu(y * x)=\mu(0) \geq \mu(x * y)$, therefore $\mu(y * x) \geq \mu(x * y)$

Hence $\mu(x * y)=\mu(y * x)$.

2- we have $\mu(x * y)=\mu((x * y) * 0)$, and by (III) from 2.1.

$$
\mu(x * y)=\mu((x * y) *(x * x)), \text {, and by the assumption }
$$

$\mu(x * y)=\mu((x * x) *(y * x))$

$$
\mu(x * y)=\mu(0 *(y * x))
$$

$\mu(x * y)=\mu(0) \geq \mu(y) \wedge \mu(z)$, because

$0 \leq z, \forall z \in X \Longrightarrow 0 * y \leq z$, and according to the definition 2.8.

$\mu(0) \geq \mu(y) \wedge \mu(z)$, hence $\mu(x * y) \geq \mu(y) \wedge \mu(z)$, that means $\mu$ is fuzzy sub-algebra of X according by 2.14 .

Theorem 3.8. Let $\mathrm{X}$ be BCI algebra and let $\mu$ be fuzzy set in $\mathrm{X}$, then $\mu$ is anti-fuzzy $\mathrm{p}$-ideal in $\mathrm{X}$ if and only if $\mu$ is 
anti-fuzzy ideal and satisfied the condition $x \leq x * y \quad \forall x, y \in X$.

Proof. Proof of the necessity of the condition exists in the reference [7]

Let us prove the opposite, let $\mu$ is anti-fuzzy ideal and satisfied the condition

$x \leq x * y \quad \forall x, y \in X$. According to definition 2.9. $\mu(0) \leq \mu(x)$.

We have $x \leq x * y \Rightarrow x * y \leq(x * y) * z$

$$
\begin{aligned}
& \Rightarrow x * y \leq(x * z) *(y * z) \\
& \Rightarrow(x * y) *((x * z) *(y * z))=0 . \text { We have } \mu \text { is anti-fuzzy ideal, therefore }
\end{aligned}
$$

$\mu(x * y) \leq \mu((x * y) *((x * z) *(y * z)) \vee \mu((x * z) *(y * z))$

$\mu(x * y) \leq \mu(0) \vee \mu((x * z) *(y * z))$, but $\mu(0) \leq \mu((x * z) *(y * z))$, so

$\mu(x * y) \leq \mu((x * z) *(y * z))$, hence

$\mu(x * y) \vee \mu(y) \leq \mu((x * z) *(y * z)) \vee \mu(y)$, but $\mu(x) \leq \mu(x * y) \vee \mu(y)$, therefore $\mu(x) \leq \mu((x * z) *(y * z)) \vee$ $\mu(y)$, hence $\mu$ is anti-fuzzy p-ideal.

\section{References}

Ahsan, J., Thaheem, A. B., \& Deeba, E. Y. (1989). On prime ideals and associated spectrum of BCK-algebras (No. IC--89/215). International Centre for Theoretical Physics.

Hong, S. M., \& Jun, Y. B. (1998). Anti fuzzy ideals in BCK-algebras. Kyungpook Mathematical Journal, 38(1), 145-145.

Jun, Y. B., \& Lee, K. J. (2011). Positive implicative vague ideal in BCK-algebras. Annals of Fuzzy Mathematics and Informatics, 1(1), 97-105. https://doi.org/10.3390/math6090149

Jun, Y., Roh, E., \& Öztürk, M. (2018). Positive Implicative Ideals of BCK-Algebras Based on Intuitionistic Falling Shadows. Mathematics, 6(9), 149.

Kaviyarasu, M., \& Indhira, K. (2017). Review on BCI/BCK-Algebras and Development. Infinite Study.

Kordi, A., \& Moussavi, A. (2007). On fuzzy ideals of BCI-algebras. Pure Mathematics and Applications, 18, 301-310.

Meng, J., \& Guo, X. E. (2005). On fuzzy ideals in BCK/BCI-algebras. Fuzzy sets and Systems, 149(3), 509-525. https://doi.org/10.1016/j.fss.2003.11.014

Mostafa, S. M., Omar, R. A. K., \& Marie, A. I. (2011). Anti fuzzy sub-implicative ideals of BCI-algebras. Journal of American Science, 7(11), 274-282.

Rosenfeld, A. (1971). Fuzzy groups. Journal of mathematical analysis and applications, 35(3), 512-517. https://doi.org/10.1016/0022-247X(71)90199-5

Solairaju, A., \& Ragavan, C. Characterizations of T-Fuzzy R-Ideals on BCI-Algebras. International Journal of Computer Applications, 975, 8887.

Zadeh, L. A. (1965). Fuzzy sets. Information and control, 8(3), 338-353. https://doi.org/10.1016/S0019-9958(65)90241-X

Zahedi, M. M., Hasankhani, A., \& Bozorgee, G. H. (1999). Some equivalent conditions of fuzzy prime dual ideals in a BCK-algebra. Scientiae Mathematicae, 2(3), 361-371.

\section{Copyrights}

Copyright for this article is retained by the author(s), with first publication rights granted to the journal.

This is an open-access article distributed under the terms and conditions of the Creative Commons Attribution license (http://creativecommons.org/licenses/by/4.0/). 